\title{
Utilização de Medicinas Alternativas e Complementares na atenção primária à saúde de Florianópolis/SC, Brasil: percepção de usuários
}

\author{
Use of Complementary and Alternative Medicine in primary \\ healthcare in Florianópolis, Santa Catarina, Brazil: user perception
}

Amanda Faqueti ${ }^{1}$

Charles Dalcanale Tesser ${ }^{1}$
${ }^{1}$ Departamento de Saúde Pública, Centro de Ciências da Saúde, Universidade Federal de Santa Catarina. Campus Universitário, Trindade. 88040-900 Florianópolis SC Brasil. amandafaqueti@gmail.com

\begin{abstract}
This is a partial analysis of the outcome of a survey looking at user perception of the institutional use of Complementary and Alternative Medicine (CAM) in Florianópolis healthcare centers. Structured interviews were applied to users treated with CAM in the locations offering this option most often, using data-based theory as a theoretical-methodological reference. For the most part, the use of CAM was proposed by basic health professionals (the operators of biomedical care), initially as a preferred alternative to biomedicine. Interviewees preferred CAM, associating it to fewer side-effects. Users mentioned enhanced dialog with professionals to negotiate treatment forms. The hypothesis emerged that CAM could be the initial treatment option in a large number of cases, saving conventional treatment for subsequent use if necessary, or in some cases as the single or complementary treatment. This enables drafting an expanded flow using CAMs in primary healthcare. New studies and institutional experience are required to investigate this hypothesis, expanding the use of CAM in an ecology of institutional care. Key words Integrative medicine, Complementary therapies, Primary healthcare
\end{abstract}

Resumo Apresenta-se análise parcial dos resultados de pesquisa que investigou as percepções de usuários sobre o uso institucional de Medicinas Alternativas e Complementares (MAC) nos centros de saúde de Florianópolis. Realizaram-se entrevistas semiestruturadas com usuários tratados com MAC nos locais com maiores ofertas, usando como referencial teórico-metodológico a teoria fundamentada em dados. Na sua maioria, o uso de MAC era proposto pelos profissionais da atenção básica (operadores do cuidado biomédico), inicialmente de forma alternativa e preferencial à biomedicina, e houve comum preferência dos entrevistados por essa forma de uso, devido aos menores efeitos colaterais atribuídos às MAC. Usuários referiram diálogo aumentado com os profissionais devido à negociação sobre as forma de tratamentos. Emergiu a hipótese de que as MAC podem ser um tratamento alternativo inicial em parte significativa dos casos, ficando o convencional como retaguarda para uso posterior se necessário, ou complementar ou único em outras situações. Isso permite esboçar um fluxo ampliado da presença das MAC na atenção primária à saúde. Novas pesquisas e experiências institucionais são necessárias para investigação dessa hipótese, que amplifica o uso das MAC numa 'ecologia de cuidados institucionais'.

Palavras-chave Medicina integrativa, Terapias complementares. Atenção primária à saúde 


\section{Introdução}

As Medicinas Alternativas e Complementares (MAC) são amplamente utilizadas em todo o mundo $^{1,2}$. Incluem práticas de cuidado e racionalidades médicas ${ }^{3}$ que não são consideradas pertencentes à medicina convencional ${ }^{4}$; e têm sido muito usadas em países de renda alta, tendo sempre sido abundantes nos outros ${ }^{5-7}$.

$\mathrm{O}$ uso das MAC nos países com amplo acesso a biomedicina tem sido atribuído a limitações (diagnósticas e terapêuticas), iatrogenias e características relacionais problemáticas do cuidado biomédico, bem como a virtudes a elas atribuídas: valorização do vínculo, escuta e abordagem ampliadas considerando várias dimensões dos doentes, melhor relacionamento com o usuário, estímulo a participação do mesmo e ao seu potencial de reequilíbrio e autocura, facilitação da significação e da experiência dos adoecimentos, centramento do cuidado na pessoa e na categoria 'saúde' e não doença ${ }^{8-13}$.

No Brasil, o governo federal as designou como práticas integrativas e complementares (PIC) e a Política Nacional de $\mathrm{PIC}^{14}$ preconiza a sua inserção no Sistema Único de Saúde (SUS), principalmente na atenção primária à saúde (APS). Todavia, não há especificação sobre o modo de inserção das MAC nos serviços. Quando presentes na APS, ora são realizadas pelos próprios profissionais que exercem o cuidado biomédico, sendo por essa característica de prática mista denominados 'híbridos' por Barros ${ }^{15}$; ora são exercidas por profissionais que atuam somente com as mesmas, comumente especialistas (como acupunturistas ou homeopatas), os quais designaremos praticantes 'exclusivos' (de MAC). Isso é relevante no Brasil, em uma perspectiva de construção da universalização do acesso ao cuidado biomédico e às MAC. A opção preferencial por praticantes exclusivos acarretaria na sua inserção na APS, somando-se aos operadores do cuidado biomédico. A opção pelos híbridos implicaria na capacitação (educação permanente) dos profissionais das equipes de saúde da família (SF) e inserção forte desse tema na sua formação.

Os trabalhos brasileiros sobre as MAC na APS abordam aspectos gerais sobre elas ${ }^{12,16-19}$, ou sua oferta pelos serviços ${ }^{20,21}$, ou a visão dos profissionais ${ }^{22,23}$. As pesquisas que enfocam a visão dos usuários da APS são escassas e mais voltadas para alguma MAC específica, investigando as motivações para sua procura, conhecimento da prática, resultados e percepções sobre o tratamento ${ }^{9,24,25}$. Essas pesquisas abordam usuários de homeopatia ou de outras práticas ${ }^{19,26}$, exercidas por adeptos exclusivos de MAC.

Em Florianópolis-SC, ocorre um processo de implantação sistemática das MAC na APS desde 2011, em que as mesmas são praticadas pelos profissionais das equipes de SF (híbridos), com competência prévia ou que são capacitados/introduzidos em alguma(s) MAC através de atividades de educação permanente ${ }^{27}$.

O objetivo deste artigo é apresentar parte dos resultados de uma pesquisa que analisou as percepções de usuários sobre suas experiências com MAC na APS de Florianópolis/SC. Tal parte versa especificamente sobre o uso institucional das MAC. Não discutiremos dados relacionados com o uso autônomo das MAC, a percepção sobre a natureza dos adoecimentos ou a atuação das MAC neles, nem os resultados obtidos, salvo alguns aspectos que contextualizam e contribuem para a compreensão do uso institucional dessas terapias.

\section{Métodos}

Realizou-se um estudo descritivo qualitativo envolvendo usuários de MAC atendidos por equipes de SF em Florianóipolis/SC. Foram selecionados os quatro centros de saúde (CS) com maior número de atendimentos em MAC, no sistema de informações municipal (InfoSaúde ${ }^{\circledR}$ ), codificados como A, B, C e D. O convite aos usuários ocorreu nas salas de espera desses serviços através de abordagem direta pela entrevistadora. Os que respondiam positivamente à pergunta sobre se estavam recebendo ou tinham recebido tratamento com alguma MAC nos últimos três meses no serviço eram convidados para uma entrevista semiestruturada, caso tivessem mais de dezoito anos de idade.

O referencial teórico-metodológico e de análise foi a Teoria Fundamentada em Dados ${ }^{28}$, que permite construir hipóteses, conceitos ou teorias a partir dos dados, sem adesão a teorias predeterminadas, e cujos métodos possuem flexibilidade para aproximação progressiva do fenômeno. Foram realizadas três rodadas de coleta de dados nos serviços, o que facilitou o aperfeiçoamento das entrevistas e o esclarecimento de dúvidas que surgiam, entre setembro/2013 e maio/2014. $\mathrm{Na}$ fase de análise não se utilizou todo potencial da teoria fundamentada em dados, já que o propósito não foi desenvolver uma nova teoria, mas auxiliar na construção de hipóteses, articulando os principais achados com outros estudos e propo- 
sições teóricas que embasam o uso institucional das MAC na APS. Isso se deveu também aos limites metodológicos e contextuais da pesquisa, com foco exclusivo nos usuários (sem investigação em prontuários e dos profissionais envolvidos) e ao modo de seleção dos participantes. Mesmo assim, optou-se por uma investigação exploratória de uma realidade relativamente desconhecida e recente, que é a ampliação das MAC induzida por educação em serviço. Poucos municípios com grande cobertura de SF e APS bem organizada optaram por implantar MAC na APS por meio de atividades educativas sistemáticas, como Florianópolis.

As entrevistas foram gravadas, transcritas e analisadas conforme ocorriam, pela mesma pesquisadora, através de codificação aberta (informações separadas, classificadas e sintetizadas de acordo com afinidades e divergências). Considerou-se haver saturação nos dados na vigésima entrevista ${ }^{29}$. Seguiu-se uma codificação axial (estabelecimento de categorias e subcategorias), e depois codificação seletiva (refinamento das principais categorias e subcategorias, algumas orientadas pelos objetivos e outras emergentes $)^{30}$.

Nove participantes eram do CS A (que fazia mais atendimentos com MAC), quatro do CS B, quatro do D e três do $\mathrm{C}$. Todos foram codificados com a letra do CS e um número identificador (A1, $\mathrm{A} 2, .$. ). As entrevistas foram realizadas em centros comunitários, residências ou salas nos CS, antes ou após os atendimentos; sua duração variou de 25 a 35 minutos. As entrevistas iniciavam, como aquecimento, pela apresentação de dez imagens impressas de MAC comuns no Brasil, questionando o conhecimento e o uso doméstico e na APS. Seguia-se um diálogo orientado por roteiro que envolvia: forma de acesso, motivos de uso, resultados obtidos, formas de utilização, diferenças entre cuidado convencional e as MAC, preferências por profissionais exclusivos de MAC ou da equipe de SF e por que. A Resolução CNS no 466 foi cumprida.

\section{Resultados e Discussão}

Foram entrevistados usuários atendidos por cinco médicos e uma enfermeira, de seis equipes de SF, dos quatro serviços, todos híbridos. Dos médicos, três tinham especialização em acupuntura e dois receberam treinamento introdutório em acupuntura promovido pela Secretaria Municipal de Saúde ${ }^{27}$, assim como a enfermeira em auriculoterapia. Apenas um dos médicos era antigo no seu serviço (mais de dez anos de prática biomédica e de MAC). Os demais eram relativamente novos no serviço ou como praticantes de MAC (menos de cinco anos). Embora a acupuntura tenha sido a prática mais utilizada, outras MAC apareceram, a maioria constando das atividades educativas da instituição: acupuntura, auriculoterapia, florais, chás (plantas medicinais) e homeopatia, associados ou não.

Todas as entrevistadas eram do sexo feminino, entre 36 e 64 anos, de ocupações variadas, principalmente donas de casa, costureiras e auxiliares de cozinha, em sua maioria católicas e evangélicas, residentes nos bairros dos CS selecionados. Realizaram no mínimo três consultas envolvendo MAC, dezesseis estavam em tratamento e quatro haviam concluído ou interrompido nos últimos três meses.

Contextualizando os dados adiante discutidos, sintetizamos que as principais razões mencionadas para a utilização das MAC foram dores corporais (músculo-esqueléticas), problemas de ansiedade, humor e estresse, geralmente associados entre si e/ou com outros adoecimentos. Todas as entrevistadas referiram melhora do problema que foi motivo de uso da MAC, ao menos temporariamente. A eficácia das MAC foi vinculada a seu modo de ação "de dentro para fora", relacionado ao estímulo do poder de autocura, por oposição ao modo de fora para dentro dos fármacos, com os quais o cuidado convencional foi identificado ${ }^{31,32}$. Tais achados convergem com outros estudos ${ }^{9,23,24,30,33,34}$ e não são discutidos aqui por fugirem do escopo deste artigo.

\section{Reconhecendo preferências invisibilizadas}

A grande maioria (15 entrevistadas) das iniciativas de uso de MAC coube aos profissionais, que durante a consulta sugeria o tratamento e já o iniciava, o que é típico e/ou quase exclusivo da prática híbrida com MAC na APS. Sete entrevistadas relataram que a proposta inicial dos profissionais era que se utilizassem as MAC de forma alternativa e anterior ao tratamento convencional: Eu tava sentindo uma dor muito forte, então eu vim conversei com ele e ele aconselhou a tentar a acupuntura [antes da medicação] (A5). Ele nunca precisou me dar remédio não, só com acupuntura já resolve (A4). Houve nesses casos a opção de valorização das MAC como preferenciais e alternativas ao cuidado convencional, inicialmente ficando o tratamento convencional como retaguarda para uso posterior se necessário. Tal uso parece ser incomum entre profissionais biomé- 
dicos. Isso poderia, talvez, ser explicado pelo tipo de profissionais híbridos que orientaram/realizaram esses tratamentos. Eles têm alguma competência em MAC por interesse pessoal - procuraram os cursos promovidos pelo município ou tinham especialização prévia (o que é regra entre os profissionais do SUS especialistas em alguma MAC $^{9,21,35}$ ). Porém, médicos de outros ambientes institucionais que usam ou indicam MAC não parecem fazê-lo da mesma forma, pois há uma tendência no ambiente institucional biomédico de uso complementar das $\mathrm{MAC}^{10,15}$; e não encontramos estudos suficientemente similares (em contexto e foco investigados) para comparação.

Tal achado é relevante porque há uma aproximação crescente dos profissionais de saúde em geral e da APS, em particular, das MAC. No mundo e no Brasil, proporções significativamente grandes nas últimas décadas de profissionais e/ou serviços de APS usam ou recomendam MAC ${ }^{1,11,36}$. Isso demanda investigações adicionais para averiguar a existência mais geral do fenômeno e sua magnitude. Um fator certamente envolvido nisso é a especificidade do ambiente de cuidado: à APS chegam adoecimentos frequentemente inespecíficos e associados, com acesso facilitado, para cuidado ao longo do tempo de todos os tipos de problemas $^{37,38}$, em que as MAC adequam-se bem (adiante retomamos este ponto).

Metade das entrevistadas também referiu preferência em utilizar as MAC antes dos tratamentos farmacológicos. Elas foram consideradas como tratamentos preferenciais tanto no uso doméstico - vou primeiro no chá, o natural, só procuro mesmo o médico quando [...] eu não aguento mesmo [B1] - como no cuidado médico - [o médico] começa com esse tratamento [MAC]..., aí, se não resolve, bora pra um químico [D1]. A utilização de MAC no ambiente doméstico de forma alternativa, complementar ou sequencial a tratamentos biomédicos é reconhecidamente prevalente ${ }^{39,40}$, permitindo uma diversidade de práticas típica da autoatenção ${ }^{31}$. Mas deve ser destacada a extensão dessa preferência das usuárias ao cuidado profissional. Não encontramos outras pesquisas com dados comparáveis. Isso parece reforçar o que Helmann ${ }^{40}$ chamou 'pluralismo terapêutico', em que há procura e valorização dos recursos biomédicos simultaneamente à intensa procura e aceitação de outros tipos de cuidados ${ }^{41}$.

Cabe registrar a diferença mais apontada entre as MAC e os fármacos, que facilita entender a comum preferência pelas primeiras: a grande presença de reações adversas no uso de medicamentos convencionais; e menos frequente nas
MAC, segundo várias entrevistadas e ampla literatura internacional': Quando a gente toma um remédio [fármaco] fica assim sentindo outras coisas que pode ser do remédio que tu tomou (C1). Eu tomava o medicamento pra dor, daí me atacava a gastrite, daí eu também tenho problema de alergia, tu fica insegura... Agora com a acupuntura e a aurículo já não tem, a acupuntura só dá coisas boas (C3). Essa visão, de várias usuárias, sobre as MAC serem tratamentos naturais inofensivos demanda também capacitação das equipes da APS sobre o tema (e sua introdução na sua formação), para que possam contribuir com orientações, pois que não são isentas de riscos (caso comum das plantas medicinais). Hoje essa competência é muita escassa e há ignorância da grande maioria dos profissionais brasileiros da APS sobre as MAC, complicada pela comum omissão dos usuários da referência ao uso autônomo das mesmas. Justamente por isso a inserção sistemática e progressiva das MAC na formação e nas práticas dos profissionais da APS seriam importantes e desejáveis, induzindo a construção e disseminação de uma expertise mínima sobre o tema nos profissionais da APS. Um maior uso institucional das MAC pelos profissionais (híbridos) da APS, operadores do cuidado biomédico, ao exigir essa expertise mínima, significaria também um mecanismo de segurança adicional em seu uso, uma vez que várias delas não têm regulamentação e/ ou legitimação científica e sendo orientadas pelos profissionais da APS, serão eles que atenderão algum efeito adverso do uso dessas práticas. .

\section{Reconhecendo diálogos anteriormente incomuns}

Foi observado que a presença das MAC é um fator facilitador de diálogo e da melhoria da relação profissional-usuário devido à negociação na construção da terapêutica: ele quer tirar as medicações [e ficar só com MAC] e eu não quero, demoro pra aceitar, já consegui diminuir, mas eu que não consigo parar (C2). Algumas entrevistadas fizeram críticas aos profissionais, por não receitarem mais medicamentos ou não solicitarem exames, mas esses relatos envolviam um acréscimo de diálogo: Ele conversou bastante, perguntou se tomava algum remédio ou não, disse que tem remédio que melhora pra uma coisa, mas piora pra outra, disse pra tentar primeiro com o chá daí se não resolvesse era pra voltar lá (B3).

São registradas na literatura insatisfações com o atendimento biomédico, dirigidas a abordagem caracterizada como mecanicista, inva- 
siva, intervencionista, fria, limitada a sintomas, centrada em doenças, com maciça interposição de aparelhagem diagnóstica na interação e pouca singularização dos tratamentos ${ }^{8,10,12,32,39,42}$. Por exemplo, Stewart et al. ${ }^{43}$ encontrou que $54 \%$ dos distúrbios percebidos pelos pacientes não eram considerados pelos médicos nas consultas. Isso indica a necessidade de melhorias nessa relação, no sentido de maior diálogo e escuta do usuário, e de melhor convergência entre ambos na interação. Devido às virtudes atribuídas às MAC mencionadas na introdução, em tese, seu exercício pelas equipes da APS teria o potencial de ampliar a abordagem dos profissionais, favorecendo uma melhor escuta e consideração de aspectos subjetivos e psicossociais. Todavia, os depoimentos colhidos referiam-se a algo diferente dessas virtudes atribuídas ao exercício das MAC: referiam-se a ação de negociação sobre o uso de tratamentos com MAC ou tratamento convencional.

Trata-se de um diálogo possível devido à existência de mais de uma opção terapêutica. Esse achado significa uma força positiva diferente na ação das MAC sobre a interação profissional-usuário nos serviços: uma virtude que não é de nenhuma MAC, mas decorre de sua presença e diferença, que demanda diálogo sobre que abordagem acionar. Isso existe sobretudo na prática profissional híbrida na APS, em que a decisão sobre o uso de biomedicina ou MAC e a avaliação/ acompanhamento do problema, com fácil acesso, são compartilhadas pela mesma dupla profissional-usuário. Sabe-se que há melhores desfechos e mais satisfação quando profissionais e usuários compartilham visões, interpretações, expectativas e decisões sobre os adoecimentos e tratamentos $^{44}$. Tal compartilhamento facilitado é uma das virtudes das MAC e também é a base de novas abordagens metodológicas biomédicas na APS, como o método clínico centrado na pessoa ${ }^{43} \mathrm{e}$ as discussões brasileiras sobre clínica ampliada ${ }^{45}$.

\section{Inserindo e organizando as MAC na APS e no SUS}

A possibilidade de integração das MAC na APS tem sido discutida fora do Brasil, abordando a atuação colaborativa entre profissionais ${ }^{46,47}$ e com foco em perspectivas de clínicos que as integram em seus serviços ${ }^{48}$. Algumas poucas pesquisas abordam a visão dos usuários ${ }^{47,49,50}$. Estes respondem variavelmente quando investigados com relação ao profissional mais adequado para realizar essas práticas. Ora desejam que o médico de família forneça a $\mathrm{MAC}^{50}$, ora que o mesmo in- forme sobre as terapias ${ }^{47,49}$, ora preferem ser encaminhados a especialistas da área ${ }^{47}$. Mas todos gostariam que os médicos da APS tivessem um papel ativo em relação às $\mathrm{MAC}^{47,50}$. No Brasil não encontramos pesquisas com esse foco.

Questionadas sobre qual a melhor forma de acessar esses tratamentos (MAC), nove entrevistadas preferiram que as MAC fossem realizadas pela equipe de SF, [...] diminui um pouco essa coisa de ficar indo de um lado pro outro passando por um clínico geral pra que ele fizesse o encaminhamento, dai evita filas e o desgaste [...] (I1). As que preferiram ser atendidas de forma especializada (oito), argumentaram que para um profissional fazer tudo é 'muita coisa' e, como há muitos pacientes, eles não conseguiriam atender a todos com ambas as abordagens, além de referirem que quando esses atendimentos são separados o profissional pode se dedicar e se aperfeiçoar em um tipo de tratamento. Tem que ter os dois separado. Ele trata mais a dor, mas não trata muito a fobia, então seria interessante que tivesse alguém pra tratar essa parte também (D4).

Dentre as entrevistadas, as usuárias de serviços especializados tratavam com MAC na APS de forma complementar e preferiam ser tratadas separadamente por especialistas exclusivos de MAC. O fato dessas usuárias estarem vinculadas a ambulatórios especializados e não se sentirem em tratamento medicamentoso na APS foi referido como influente na sua preferência: nunca consultei com o Dr. com outro tratamento, porque ainda me trato com os meus médicos [especialistas focais], faço acompanhamento [com eles], então aqui [APS] eu venho pra medir a pressão e agora a acupuntura pro ciático (A5). Por outro lado, se a gravidade de uma situação pode demandar cuidados especializados biomédicos, isso permite entender a sua preferência por praticantes exclusivos (e especializados quando existentes) de MAC: seus problemas também mereceriam ser explorados em profundidade por uma MAC, devido à maior necessidade/gravidade clínica. Assim, esses tratamentos, como a biomedicina, além de estarem acessíveis na APS, poderiam ter lugar também na atenção especializada. Esta é composta de serviços ambulatoriais, hospitalares e equipes de apoio à APS, como os Núcleos de Apoio à Saúde da Família $\left(\mathrm{NASFs}^{51}\right.$ ), os quais podem ter profissionais homeopatas e acupunturistas (praticantes exclusivos). Estes poderiam ser referência especializada para casos graves referenciados, explorando com profundidade estas MAC.

Os NASFs devem exercer cuidado especializado e também atuar como suporte técnico (edu- 
cação permanente, discussão de casos, regulação) personalizado para um grupo de equipes de SF. Sua atuação é de uma equipe de profissionais especializados (não generalistas) de inserção excelente, pois próxima das equipes da APS e por elas coordenados. Isso parece apropriado para várias MAC, especialmente as racionalidades médicas vitalistas ${ }^{2}$, como a homeopatia, a medicina tradicional chinesa/acupuntura e a medicina antroposófica. Essa consideração reforça a desejabilidade das MAC estarem integradas nas equipes de SF (profissionais híbridos) e também, em serviços especializados com matriciamento, exercidas por praticantes exclusivos.

\section{Visualizando os fluxos de uso institucional das MAC na APS e no SUS}

As preferências das usuárias, mencionadas em parte das entrevistas, e também dos profissionais híbridos da APS em iniciar o cuidado com MAC antes dos tratamentos convencionais, associadas com o maior diálogo derivado da sua presença, sustentam a hipótese de uma possível inversão de sentido da complementaridade das MAC na APS Nesse contexto, em uma parte das situações, as MAC teriam certa primazia na APS e seriam usadas como tratamento alternativo inicial preferencial, sendo complementadas, quando necessário, pela biomedicina. A farmacoterapia seria, aí, utilizada de forma complementar, nas situações resistentes a cuidados iniciais com MAC e/ou mais graves, além de ser preferida em outra parte dos casos, ou complementada por alguma MAC. Assim como no ambiente familiar e comunitário em que as MAC são abundantes, apesar da (bio)medicalização crescente da sociedade $^{52}$, seria possivelmente desejável e adequado tecnologicamente essa inversão de preferência no ambiente institucional da APS em parte dos casos, quando houver abertura dos usuários, gerando o uso alternativo inicial.

Um argumento a favor dessa hipótese é que há convergência entre o tipo de adoecimento que chega na APS, frequentemente inicial, pouco enquadrável na nosologia biomédica e comumente envolvendo vários problemas e as virtudes atribuídas às MAC (sintetizadas na introdução). Tal convergência é suficientemente forte para que McWhinney ${ }^{38}$, em clássico da Medicina de Família e Comunidade, reconheça a APS como o lócus privilegiado de inserção das MAC nos sistemas de saúde; e daí decorre, provavelmente, que 70\% da sua presença "espontânea" no SUS se dê na atenção básica ${ }^{14}$.
Outro argumento forte é a iatrogenia frequente e o comum excesso de intervenções do cuidado biomédico, tão significativo que deu origem na Medicina de Família e Comunidade ao conceito e prática da prevenção quaternária: identificar pessoas em risco de sobremedicalização e dano iatrogênico e oferecer-lhes alternativas eticamente aceitáveis ${ }^{53}$. Trata-se de operacionalizar o princípio do primun no nocere, para além do discurso; o que exige saberes e habilidades específicos, voltados para a proteção do usuário da tendência atual de hipermedicalização diagnóstica e terapêutica, curativa e preventiva ${ }^{54}$. O uso criterioso e preferencial de MAC na APS poderia ser considerado em muitos casos uma ação de prevenção quaternária: um filtro intermediário protetor (com supostos menores efeitos iatrogênicos) a se interpor entre o primeiro, dos cuidados familiares e autônomos, sabidamente importante ${ }^{39,55}$, em que as MAC já são abundantes, e o biomédico, exercido pelos profissionais da APS $^{55,56}$.

A articulação dos resultados apresentados permite esboçar um modelo hipotético de fluxos assistenciais institucionais, incorporando as MAC de modo coerente com os ambientes de cuidado e os tipo de adoecimentos, que se constitui em proposta de concretização da pluralidade de cuidados no SUS, como hipótese emergente desta pesquisa, ilustrada na Figura 1.

Nota-se na Figura 1 que na APS as MAC entrariam em dois fluxos, praticadas por profissionais híbridos: como cuidado preferencial inicial (momentaneamente alternativo, que pode ser suficiente ou não) em uma parte dos casos, exercendo uma função filtro anterior ao cuidado biomédico; e como cuidado complementar, noutra parte dos casos. Um terceiro grupo de usuários receberia apenas cuidado biomédico (hoje muito mais comum). As MAC também poderiam entrar na atenção especializada, exercidas então por especialistas (ou praticantes) exclusivos. Estão articulados coerentemente nestes fluxos os nossos resultados e outros saberes consolidados: a aproximação de profissionais da APS das MAC e a sua grande aceitação e uso pelas populações; os frequentes efeitos adversos da farmacoterapia; os tipos indiferenciados, combinados e complexos de adoecimentos trazidos à APS; o acesso fácil nesta última; as virtudes das MAC; a possibilidade de maior segurança no seu uso pelos profissonais híbridos dentro da longitudinalidade; e a desejabilidade de maior diálogo nas interações profissionais-usuários sobre os tratamentos e o uso de MAC, além das virtudes atribuídas às MAC sintetizadas na introdução. 


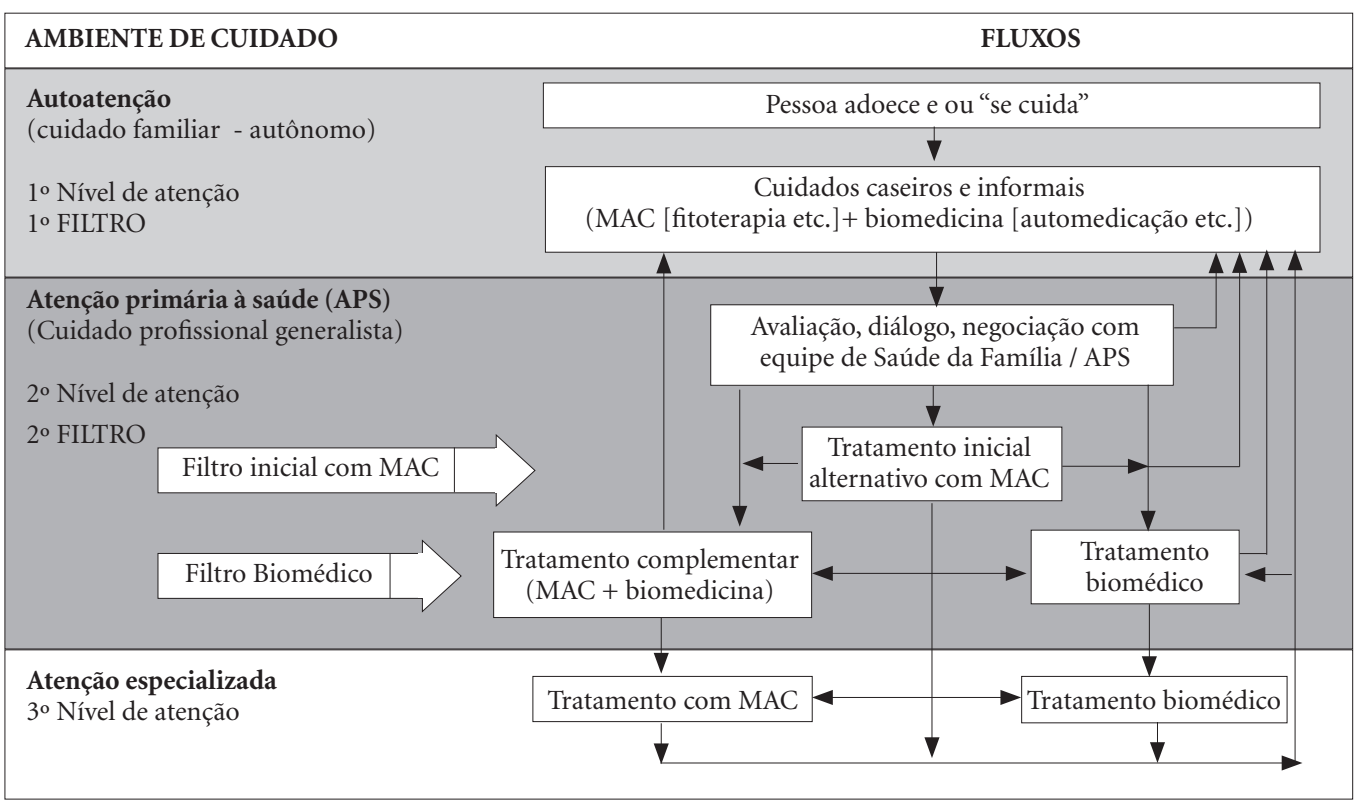

Figura 1. MAC na ecologia e na organização dos cuidados.

As setas significam possibilidades de fluxos. $\mathrm{MAC}=$ medicina alternativa e complementar.

Fonte: elaborado pelos autores a partir dos resultados e inspirado em Green et al..$^{39} \mathrm{e}$ Menendez ${ }^{33}$.

A hipótese levantada suscita questões complexas, aqui apenas apontadas. Por exemplo, comumente há exigência de evidências científicas sobre segurança e eficácia para tratamentos não convencionais, e para boa barte das MAC ou isso não existe, ou é controverso ou está pouco desenvolvido. Há algumas evidências recentes de bons resultados do uso de algumas MAC na APS por profissionais híbridos: menores custos hospitalares e com medicamentos e menor mortalidade ${ }^{57}$. Embora cresçam os estudos sobre várias MAC, as racionalidades médicas vitalistas, pelo menos, poderiam ser reconhecidas e legitimadas mesmo sem consensuais evidências biomédicas sustentando-as, devido, em parte, à sua legitimidade, ampla aprovação e eficácia socialmente reconhecida, com poucos riscos comparativamente aos tratamentos convencionais; e em parte devido à incomensurabilidade (em relação à biomedicina) dos seus saberes e técnicas imersos em paradigmas próprios, com concepções distintas de adoecimento e ação curativa. Por outro lado, como já mencionamos, a segurança de seu uso é aumentada pelo próprio contexto institucional, que proporciona acompanhamento biomédico na APS e nos casos mais graves, provavelmente em serviços especializados, o que certamente funciona como mecanismo protetor dos usuários (de possíveis efeitos adversos das MAC).

Doutra parte, há conflitos políticos envolvidos. A corporação médica no Brasil tende a ser autocentrada e resistente ao reconhecimento das MAC ou de várias delas ${ }^{10}$; por outro lado, é agressivamente monopolizadora das práticas uma vez aceitas (o conflito jurídico sobre a acupuntura no Brasil é exemplo típico). Tais problemas não devem impedir sua presença; ao contrário, são em geral atenuados pela maior experimentação institucional das MAC. Além disso, para várias práticas dentro das MAC não há conflitos significativos, sobretudo as de promoção da saúde ${ }^{16} \mathrm{e}$ as coletivas.

Também deve ser considerado nesse aspecto político, o que Santos e Meneses ${ }^{58}$ chamam de linhas abissais, típicas do pensamento moderno: linhas imaginárias para além das quais há um relacionamento com o que existe em termos de apropriação e/ou violência. No caso da saúde, 
o lado de cá das linhas abissais seria composto pelos saberes e práticas em saúde profissionalizados e cientificizados instituídos. A maioria das MAC estariam para lá de uma linha abissal invisível e assim sua consideração é pensada apenas em termos de apropriação (matérias prima para a biociência/biomedicina) ou violência (combate, desqualificação e supressão). Para além dessas possibilidades, hoje realidades, outro significado de nossa hipótese é pensá-la como um exercício de 'sociologia das ausências e das emergências', visando construir uma ecologia de saberes ${ }^{59}$. Sintetizando ao extremo, tal proposta parte da constatação de que a experiência social é muito mais ampla e variada do que aquilo que a tradição científica conhece e considera importante, incluindo o cuidado à saúde. Nela, objetiva-se transformar objetos tidos como impossíveis (incompreensíveis, irrelevantes, pré-modernos uso de muitas MAC) em possíveis; e, com base neles, transformar ausências em presenças, centrando-se nos fragmentos da experiência social não focalizados e não valorizados pela perspectiva hegemônica.

A sociologia das ausências visa substituir a monocultura do saber científico (lado de cá das linhas abissais) por uma ecologia de saberes, enquanto a sociologia das emergências é a investigação das alternativas que cabem no horizonte das possibilidades concretas. Nossa hipótese pode ser considerada uma alternativa possível à monocultura de saber biomédico na APS (e no SUS). Trata-se de experiência real vivida por parte dos profissionais e usuários da APS em um município, porém invisível e irrelevante se não visibilizada e discutida. Nesse sentido, é defensável o reconhecimento e a possiblidade de uma gama de cuidados com MAC (plantas medicinais, práticas corporais, meditativas e energéticas, homeopatia, acupuntura, antroposofia, massagem) de relativo baixo risco que poderiam ganhar maior espaço como recursos de cuidado (inclusive de primeira linha, em parte dos casos) na APS e no SUS.
Provavelmente, a maior limitação desta pesquisa foi o viés de seleção dos participantes, a maioria na continuidade do tratamento com MAC, o que pressupõe aceitação e alguma satisfação com a terapêutica. Por isso, nossos dados empíricos não podem ser generalizados e nem se pode dimensionar a partir deles que proporção dos usuários optaria pelo uso alternativo inicial descrito. Por outro lado, eles permitem uma generalização hipotética cujos significados teóricos, institucionais e sociais são relevantes no contexto de emergências e possibilidades ${ }^{59}$ acima mencionado, merecendo pesquisas adicionais.

\section{Considerações finais}

Para além do uso alternativo ou complementar, encontramos em parte das entrevistadas um uso alternativo inicial preferencial das MAC por profissionais e usuárias da APS, o qual, articulado com outros achados, permitiu a proposição de um fluxo assistencial includente das MAC na APS e no SUS. Tal fluxo fortalece a ideia da presença das MAC praticadas por profissionais híbridos (praticantes do cuidado biomédico) na APS, supondo movimento progressivo de capacitação profissional em MAC e viabilizando abertura da APS para uma pluralidade institucional de cuidados. A coerência com os poucos estudos similares e a ampla aceitabilidade e uso das MAC pelos usuários (e progressiva pelos profissionais) sugerem que esse uso alternativo inicial seja significativo e adequado para uma parcela dos casos a dimensionar. Caso essa hipótese seja consistente e coerente com novos estudos e experimentações institucionais, vai gerar desafio adicional ao já posto na construção da APS brasileira: além da sua expansão e da qualificação de seus profissionais (biomédica), haverá necessidade de expansão importante da quase inexistente formação atual em MAC para os graduandos e profissionais em serviço. 


\section{Colaboradores}

A Faqueti trabalhou na concepção e na análise e interpretação dos dados e CD Tesser na metodologia, análise e redação final do artigo.

\section{Referências}

1. Organización Mundial de la Salud (OMS). Estrategia de la OMS sobre medicina tradicional 2014-2023. Genebra: OMS; 2013.

2. Organização Panamericana de Saúde. Declaração de Alma-Ata: Conferencia Internacional sobre Cuidados Primários de Saúde. Alma-Ata, 1978. [acessado 2014 Abr 10]. Disponível em: http://www.opas.org.br/promoçao/uploadarq/alma-ata.pdf

3. Luz MT, Barros NF, organizadores. Racionalidades médicas e práticas integrativas em saúde: estudos teóricos e empíricos. Rio de Janeiro: UERJ/IMS/LAPPIS; 2012.

4. National Center of Complementary and Alternative Medicine. What is complementary and alternative medicine? [Internet]. Bethesda: NCCAM; 2011. [acessado 2012 Abr 05]. Disponível em: http://nccam.nih.gov/ health/whatiscam/\#1

5. World Health Organization (WHO). The World Medicines Situation 2011 Tradicional Medicines: Global Situation, Issues and Challenges. Geneva: WHO; 2011.

6. Nogales-Gaete J. Medicina alternativa y complementaria. Rev Chil Neuro-Psquiatria 2004; 42(4):243-225.

7. Spadacio C, Castellanos MEP, Barros NF, Alegre SM, Tovey P, Broom A. Medicinas alternativas e complementares: uma metassíntese. Cad Saude Publica 2010; 26(1):7-13.

8. Souza EFAA, Luz MT. Bases socioculturais das práticas terapêuticas alternativas. Hist. cienc. saude-Manguinhos 2009; 16(2):393-405.

9. Salles SAC, Ayres JRCM. A consulta médica homeopática: examinando seu efeito em pacientes da atenção básica. Interface (Botucatu) 2013; 17(45):315-326.

10. Luz MT. Cultura contemporânea e medicinas alternativas: novos paradigmas em saúde no século XX. Physis 2005; 15(Supl.):145-176.

11. Levin JS, Jonas WB, organizadores. Tratado de medicina complementar e alternativa. São Paulo: Manole; 2001.

12. Tesser CD, Barros NF. Medicalização social e medicina alternativa e complementar: pluralização terapêutica do sistema único de saúde. Rev Saude Publica 2008; 42(5):914-920.

13. Andrade JT. Medicina alternativa e complementar: experiência, corporeidade e transformação. Salvador, Fortaleza: UFBA, EdUECE; 2006.

14. Brasil. Portaria no 971, de 3 de maio de 2006. Dispõe sobre a integralidade da atenção como diretriz do SUS e aprova a Política Nacional de Práticas Integrativas e Complementares (PNPIC) no Sistema Único de Saúde. Diário Oficial da União 2006; 3 maio.

15. Barros NF. Medicina complementar: uma reflexão sobre o outro lado da prática médica. São Paulo: Annablume/ FAPESP; 2000.

16. Tesser CD. Práticas Complementares, racionalidades médicas e promoção da saúde: contribuiç̃oes pouco exploradas. Cad Saude Publica 2009; 25(8):1732-1742.

17. Andrade JT, Da Costa LFA. Medicina complementar no SUS: práticas integrativas sob a luz da antropologia médica. Saúde Soc 2010; 19(3):497-508.

18. Tesser CD, Sousa IMC. Atenção primária, atenção psicossocial, práticas integrativas e complementares e suas afinidades eletivas. Saúde Soc 2012; 21(2):336-350.

19. Tesser CD, Luz MT. Racionalidades médicas e integralidade. Cien Saude Colet 2008; 13(1):195-206.

20. Sousa IMC, Vieira ALS. Serviços Públicos de saúde e medicina alternativa. Cien Saude Colet 2005; 10(Supl. 1):255-266.

21. Sousa IMC, Bodstein RCDA, Tesser CD, Santos FDADS, Hortale VA. Práticas integrativas e complementares: oferta e produção de atendimentos no SUS e em municípios selecionados. Cad Saude Publica 2012; 28(11):2143-2154.

22. Nagai SC, Queiroz MS. Medicina Complementar e Alternativa na Rede Básica de Serviços de Saúde de Campinas: uma aproximação qualitativa. Cien Saude Colet 2011; 16(3):1793-1800.

23. Thiago SC, Tesser CD. Percepção de médicos e enfermeiros da Estratégia de Saúde da Família sobre terapias complementares. Rev Saude Publica 2011; 45(2):249257.

24. Santanna C, Hennington EA, Junges J. Prática médica homeopática e a integralidade. Interface (Botucatu) 2008; 12(25):233-246. 
25. Monteiro DA, Iriart JAB. Homeopatia no Sistema Único de Saúde: Representações dos usuários sobre o tratamento homeopático. Cad Saude Publica 2007; 23(8):1903-1912.

26. Neves LCP, Selli L, Junges R. A integralidade na Terapia Floral e a viabilidade de sua inserção no Sistema Único de Saúde. Mundo saúde 2010; 34(1):57-64.

27. Santos MC, Tesser CD. Um método para a Implantação e Promoção de acesso às Práticas Integrativas e Complementares na Atenção Primária à Saúde. Cien Saude Colet 2012; 17(11):3011-3023.

28. Strauss A, Corbin J. Pesquisa qualitativa: técnicas e procedimentos para o desenvolvimento de teoria fundamentada. $2^{\mathrm{a}}$ ed. Porto Alegre: Artmed; 2008.

29. Fontanella BJB, Ricas J, Turato ER. Amostragem por saturação em pesquisas qualitativas em saúde: contribuições teóricas. Cad Saude Publica 2008; 24(1):1727.

30. Silva EDC, Tesser CD. Experiência de pacientes com acupuntura no Sistema Único de Saúde em diferentes ambientes de cuidado e (des) medicalização social. Cad Saude Publica 2013; 29(11):2186-2196.

31. Almeida ELV. As Razões da Terapêutica: empirismo e racionalismo na medicina. Niterói: EDUFF; 2002

32. Camargo Júnior KR. Biomedicina, ciência \& saber: uma abordagem crítica. São Paulo: Hucitec; 2003.

33. Kessler RC, Davis RB, Foster DF, Van Rompay MI, Walters EE, Wilkey SA, Kaptchuk TJ, Eisenberg DM. Long-Term Trends in the Use of Complementary and Alternative Medical Therapies in the United States Ann Intern Med 2001;135(4):262-268.

34. Helmann CG. Cultura, saúde e doença. $5^{\text {a }}$ ed. Porto Alegre: Artmed; 2009.

35. Queiroz MS. O itinerário rumo às medicinas alternativas: uma análise em representações sociais de profissionais da saúde. Cad Saude Publica 2000; 16(2):363375.

36. Dobson R. Half of general practices offer patients complementary medicine. BMJ 2003; 327(7426):1250. [acessado 2010 Mar 29]. Disponível em: http://www. bmj.com/cgi/content/full/327/7426/1250-f

37. Starfield B. Atenção Primária: Equilíbrio entre Necessidades de Saúde, serviços e teconologia. Brasília: Unesco, Brasil. Ministério da Saúde; 2002.

38. McWhinney IR. Manual de medicina de família e comunidade. $3^{\mathrm{a}}$ ed. Porto Alegre: Artmed; 2010

39. Menéndez E. Modelos de atención de los padecimientos: de exclusiones teóricas y articulaciones prácticas. Cien Saude Colet 2003; 8(1):185-208.

40. Helmann CG. Cultura, saúde e doença. $5^{\text {a }}$ ed. Porto Alegre: Artmed; 2009.

41. Le Fanu J. The rise and fall of modern medicine. London: Carroll \& Graf; 2000.

42. Stewart MA, McWhinney IR, Buck CW. The doctor-patient relationship and its effect upon outcome. J R Coll Gen Practioners 1979; 29(199):77-82.

43. Stewart M, Brown JB, Weston WW, McWhinney IR, McWhinney CL, Freeman TR. Medicina centrada na pessoa: transformando o método clínico. $2^{\text {a }}$ ed. Porto Alegre: Artmed; 2010.

44. Stewart M, Brown JB, Donner A, McWhinney IR, Oates J, Weston WW, Jordan J. The impact of patient-centered care on outcomes. J Fam Pract 2000; 49(9):796-804.

45. Campos GWS. Saúde Paidéia. São Paulo: Hucitec; 2003.
46. Chung VCH, Ma PH, Hong LC, Griffiths SM. Organizational determinants of interprofessional collaboration in integrative health care: systematic review of qualitative studies. PloS one 2012; 7(11):e50022.

47. Jong M, Van de Vijver L, Busch M, Fritsma J, Seldenrijk R. Integration of complementary and alternative medicine in primary care: What do patients want? Patient Educ Couns 2012; 89(3):417-422.

48. Shuval JT, Gross R, Ashkenazi Y, Schachter L. Integrating CAM and Biomedicine in Primary Care Settings Physicians' Perspectives on Boundaries and Boundary Work. Qual Health Res 2012; 22(10):1317-1329.

49. Ben-Arye E, Frenkel M, Klein A, Scharf M. Attitudes toward integration of complementary and alternative medicine in primary care: perspectives of patients, physicians and complementary practitioners. Patient Educ Couns 2008; 70(3):395-402.

50. Frenkel M, Ben Arye E, Carlson C, Sierpina V. Integrating complementary and alternative medicine into conventional primary care: the patient perspective. Explore (NY) 2008; 4(3):178-186.

51. Brasil. Portaria GM no 154, de 24 de janeiro de 2008. Cria os Núcleos de Apoio à Saúde da Família - NASF. Diário Oficial da União 2008; 25 jan.

52. Clarke AE, Shim j. Medicalization and biomedicalization revisited: technoscience and transformations of health, illness and American medicine. In: Pescosolido BA, Martin JK, McLeod JD, Rogers A, editors. Handbook of the Sociology of Health, Illness, and Healing. New York: Springer; 2011. p. 173-199.

53. Kuehlein T, Sghedoni D, Visentin G, Gérvas J, Jamoulle M. Prevenção quaternária, uma tarefa do clínico geral. 2009. [acessado 2014 Abr 10]. Disponível em: http://www.primary-care.ch/docs/primarycare/archiv/ de/2010/2010-18/2010-18-368_ELPS_port.pdf

54. Jamoulle M. Prevenção quaternária: a propósito de um desenho. Rev Port Med Geral Fam 2012; 28(6):398-399.

55. Green LA, Yawn BP, Lanier D, Dovey SM, Novo W. The ecology of medical care revisited. $N$ Engl J Med 2001: 344(26):2021-2025.

56. Gérvas J, Pérez Fernández M. El fundamento científico de la función de filtro del médico general. Rev. bras. epidemiol. 2005; 8(3):205-218.

57. Kooreman P, Baars EW. Patients whose GP knows complementary medicine tend to have lower costs and live longer. Eur J Health Econ 2012; 13(6):769-776.

58. Santos BS, Meneses MP, organizadores. Epistemologias do Sul. São Paulo: Cortez; 2010.

59. Santos BS. Para uma sociologia das ausências e uma sociologia das emergências. Revista Crítica de Ciências Sociais 2002; 63:237-280.

Artigo apresentado em 03/09/2015

Aprovado em 22/08/2016

Versão final apresentada em 24/08/2016 